\title{
Diagnostic value of urine sTREM-1 for sepsis and relevant acute kidney injuries: a prospective study
}

Long-xiang Su ${ }^{1,3}$, Lin Feng ${ }^{1}$, Jie Zhang ${ }^{1}$, Yong-jiu Xiao ${ }^{1}$, Yan-hong Jia ${ }^{1}$, Peng Yan ${ }^{1}$, Dan Feng ${ }^{2}$ and Li-xin Xie ${ }^{1^{*}}$

\begin{abstract}
Introduction: We explored the diagnostic value of a urine soluble triggering receptor expressed on myeloid cells-1 (sTREM-1) for early sepsis identification, severity and prognosis assessment, and for secondary acute kidney injury (AKI). We compared this with white blood cell (WBC) counts, serum C-reactive protein (CRP), serum procalcitonin $(\mathrm{PCT})$, urine output, creatinine clearance $(\mathrm{CCr})$, serum creatinine $(\mathrm{SCr})$, and blood urea nitrogen (BUN).

Methods: We enrolled 104 subjects admitted to the ICU: 16 cases with systemic inflammatory response syndrome (SIRS); 35 with sepsis and 53 with severe sepsis. Results for urine sTREM-1, WBC, serum CRP and serum PCT were recorded on days 1, 3, 5, 7, 10, and 14. For 17 sepsis cases diagnosed with secondary AKl, comparisons between their urine sTREM-1, urine output, CCr, SCr and BUN at diagnosis and $48 \mathrm{~h}$ before diagnosis were made.

Results: On the day of admission to the ICU, and compared with the SIRS group, the sepsis group exhibited higher levels of urine sTREM-1 and Acute Physiologic Assessment and Chronic Health Evaluation II (APACHE II) scores $(P<0.05)$. Areas under the curve (AUC) shaped by the scores were $0.797(95 \% \mathrm{Cl} 0.711$ to 0.884$)$ and 0.722 (95\% Cl 0.586 to 0.858 ), respectively. On days $1,3,5,7,10$, and 14, urine sTREM-1, serum PCT and WBC levels registered higher in the severe sepsis group in contrast to the sepsis group $(P<0.05)$. Urine sTREM-1 and serum PCT levels continuously increased among non-survivors, while WBC and serum CRP levels in both groups declined. For 17 patients with AKI, urine sTREM-1, SCr and BUN levels at $48 \mathrm{~h}$ before AKI diagnosis were higher, and $\mathrm{CCr}$ level was lower than those for non-AKI subjects $(P<0.05)$. AUC for urine sTREM-1 was $0.922(95 \% \mathrm{Cl} 0.850$ to 0.995), the sensitivity was 0.941 , and the specificity was 0.76 (based on a cut-off point of $69.04 \mathrm{pg} / \mathrm{ml}$ ). Logistic regression analysis showed that urine STREM-1 and severity were risk factors related to AKI occurrence.
\end{abstract}

Conclusions: Besides being non-invasive, urine sTREM-1 testing is more sensitive than testing WBC, serum CRP, and serum PCT for the early diagnosis of sepsis, as well as for dynamic assessments of severity and prognosis. It can also provide an early warning of possible secondary AKI in sepsis patients.

Trial Registration: ClinicalTrial.gov identifier NCT01333657

Keywords: urine, soluble triggering receptor expressed on myeloid cells-1(STREM-1), sepsis, severity, prognosis, acute kidney injury (AKI), sensitivity, specificity

\footnotetext{
* Correspondence: xielx@263.net

'Department of Respiratory Diseases, Chinese PLA General Hospital, 28

Fuxing Rd, Beijing, 100853, China

Full list of author information is available at the end of the article
}

C Biomed Central

(C) 2011 Su et al.; licensee BioMed Central Ltd. This is an open access article distributed under the terms of the Creative Commons Attribution License (http://creativecommons.org/licenses/by/2.0), which permits unrestricted use, distribution, and reproduction in any medium, provided the original work is properly cited. 


\section{Introduction}

Sepsis is a major factor contributing to ICU admissions and patient deaths. A recent epidemiologic study in North America found that the incidence of sepsis was 3\% in the total population and that the mortality rate for septic shock patients was as high as 50\% [1]. Because of its rapid progression, the disease might, within a relatively short period of time, lead to secondary multiple organ dysfunction syndrome (MODS) and endanger a patient's life [2]. Acute kidney injury (AKI) is well known to be associated with longer length of stay, morbidity and mortality in adults [3,4]. Sepsis-related kidney failure occurs in about $19 \%$ of sepsis patients. This incidence may reach $23 \%$ among severe sepsis patients and is 51\% among those with positive blood cultures, with a mortality rate of $70 \%$, much higher than among patients who are free from these complications [5]. Clinical practice has shown that for patients with severe sepsis and septic shock, an early, effective intervention can clearly improve prognosis and reduce mortality [6]. For this reason, it is clinically important to identify indicators that can be used for the early diagnosis and prognosis of sepsis and its related AKI.

The triggering receptor expressed on myeloid cells-1 (TREM-1) is a recently discovered member of the immunoglobulin superfamily of receptors that is expressed on polymorphonuclear granulocytes and mature monocytes. Bacterial or fungal infections may induce its expression. STREM-1 is a soluble form of TREM-1 that may be released into body fluids upon the up-regulated expression of TREM-1 [7]. An increasing number of studies indicate that there are increased levels of sTREM-1 in body fluid samples for the following diseases and conditions: sepsis, pneumonia, pleural effusion, septic arthritis, meningitis, peritonitis and uterine cavity infection [8-14]. This suggests that sTREM-1 may be a valuable diagnostic indicator for making distinctions between infectious and non-infectious diseases. It has also been found that infectious shock patients have high levels of serum sTREM-1 that are closely related to the severity of infection $[15,16]$. With regard to sepsis prognosis, dynamic changes in serum sTREM-1 may provide warnings concerning survival or fatality $[17,18]$.

Inflammation is now believed to play a major role in the pathophysiology of AKI $[19,20]$. It is hypothesized that the initial insult results in morphological and/or functional changes in vascular endothelial cells and/or in tubular epithelium in sepsis models $[21,22]$. Then, leukocytes including neutrophils, macrophages, natural killer cells, and lymphocytes infiltrate into the injured kidneys and induce the generation of inflammatory mediators [23,24]. Whether urine sTREM-1 could be detected and its significance in sepsis and AKI have not been reported yet.

The present study focused on the value of urine sTREM-1 for sepsis identification, severity and prognosis assessments, and potential sepsis-related AKI. Comparisons are also made among sTREM-1, WBC counts, serum CRP, serum procalcitonin (PCT), urine output, creatinine clearance $(\mathrm{CCr}), \mathrm{SCr}$, and blood urea nitrogen (BUN) among sepsis patients, in an effort to define relevant diagnostic values.

\section{Materials and methods \\ Study subjects}

All subjects were selected from among inpatients who were hospitalized between March 2010 and March 2011 in the Respiratory ICU, Surgical ICU and Emergency ICU of Chinese People's Liberation Army (CPLA) General Hospital. Based on the 1991 ACCP/SCCM Sepsis Directory [25] and the diagnosis criteria advanced by the 2001 International Sepsis Definition Conference [26], the subjects were divided into a systemic inflammatory response syndrome (SIRS) group and a sepsis group, with the occurrence of infection as the norm for division. Based on the severity of their conditions, the latter was sub-divided into a sepsis group and a severe sepsis group (severe sepsis and septic shock). Sepsis patients were also divided into a survivor group and a non-survivor group, with a 28 -day survival as the dividing line. This study focuses on dynamic changes of different indicators. For this, all the patients involved stayed in ICUs $\geq 14$ days or died within 14 days.

The 2006 Acute Kidney Injury Network (AKIN) defines AKI as different degrees of abnormal kidney structure and function as well as abnormal kidney damage signs lasting not more than three months, and manifested clinically by blood, urine and tissue tests and imaging studies [27], characterized by a $48 \mathrm{~h}$ absolute increase in serum creatinine $(\mathrm{SCr}) \geq 26.4 \mu \mathrm{mol} / \mathrm{L}(0.3$ $\mathrm{mg} / \mathrm{dL}$ ) or a percentage increase $\geq 50 \%$, or $\mathrm{a} \geq 6 \mathrm{~h}$ decline in urine output to $\leq 0.5 \mathrm{ml} / \mathrm{kg} \cdot \mathrm{h}$.

Patients excluded: (1) were younger than 18 years of age; (2) suffered from anuria; (3) contracted acquired immunodeficiency syndrome; (4) had reduced polymorphonuclear granulocyte counts $\left(<500 \mu \mathrm{L}^{-1}\right)$; (5) were receiving dialysis treatment for chronic kidney disease; (6) died within $24 \mathrm{~h}$ after admission into the ICU, or refused to participate in the study, or declined treatment during the period of observation. Patients or their family members were fully informed of the study details and signed informed consent forms of their own accord. This study was approved by the Ethics Committee of the CPLA General Hospital (project No.20090923-001) and was registered with the U.S. National Institutes of Health Clinical Trials Register (NCT 01333657).

\section{Data collection}

Upon admission into the ICU, the following items were recorded for each patient: age, gender, chief complaints 
for admission, symptoms, APACHE II scores, Sequential Organ Failure Assessment (SOFA) scores, white blood cell (WBC) counts, serum c-reactive protein (CRP), PCT, urine sTREM-1, CCr, SCr, BUN, urine output, mechanical ventilation, continuous renal replacement treatment (CRRT), AKI, etiological factors and underlying diseases. A record was also kept of 28-day survivals.

\section{Assays}

Within $24 \mathrm{~h}$ (the first day of study) after ICU admission, blood and urine samples were collected. Tests on intravenous blood and urine samples were repeated on the mornings of days $3,5,7,10$, and 14 . Blood was centrifuged at 3,000 rpm for 15 minutes, and urine at 2,000 rpm for 5 minutes. The supernatants were transferred to Eppendorf tubes and stored at $-80^{\circ} \mathrm{C}$. All the specimens were re-numbered before the experiment. We saw to it that each step was blind to the staffers involved. sTREM-1 was examined by a double antibody sandwich ELISA (Quantikine Human TREM-1 Immunoassay ELISA Kit, R \& D Systems, Minneapolis, MN, USA, product number DTRM10B); CRP, by scattering turbidimetry (Cardio Phase hsCRP, Siemens, Munich, Germany); PCT, by enzyme-linked fluorescence analysis (ELFA, VIDAS BRAHMS PCT kit, BioMérieux, Marcy-l'Étoile, France) and SCR and BUN, by an enzymatic method (CREA plus \& UREA/BUN reagent, Roche Modular, Mannheim, Germany). ELISA was performed in duplicate and other assays were done in strict accordance with the manufacturers' instructions.

\section{Statistical analysis}

Results for continuous variables with normal distributions, including age, WBC counts, urine output, $\mathrm{CCr}$, APACHE II scores and SOFA scores are given as means \pm standard deviations (SD). Student's $t$-test was used to compare means between two groups. Analysis of variance (ANOVA) was used to compare means among multiple groups. Results for continuous variables that were not normally distributed, including STREM-1, CRP, PCT, SCr and BUN, are given as medians $\left(25^{\text {th }}\right.$ and $75^{\text {th }}$ percentiles) and were compared using nonparametric tests. Results for qualitative variables were expressed as percentages and compared between groups using a Chi-square test. Areas under receiver operating characteristic curves were used to evaluate how well the model distinguished sepsis patients from SIRS patients, and AKI patients from non-AKI patients. Multivariate analysis used a logistic regression model to estimate the odds ratio for AKI and the 95\% confidence interval (CI). Stepwise and forward selection procedures were used to iteratively select variables possibly related to AKI. To be entered into this model, a $P<0.05$ from univariate analysis was required. Risk factors related to disease were explored using multivariate logistic regression. Statistical analyses were conducted by SPSS 16.0 (SPSS, Chicago, IL, USA) and a two-tailed $P<0.05$ was considered significant.

\section{Results}

\section{Subject characteristics}

A total of 205 patients with SIRS were involved in the study. Of these, 21 patients were later excluded according to the exclusion criterion. Of the remaining 184 patients, 56 were barred from analysis for a less-than14-day ICU stay (because of later admission or a transfer out of ICUs within 14 days, which fails the requirements of continuous observation). Later we sifted out 24 more owing to the limited concentration range of R\&D sTREM-1 assay kit. Finally, this study had 104 subjects involved, which included 16 cases of SIRS and 88 cases of sepsis. According to the study guidelines, sepsis patients were further divided into a sepsis group (35 cases) and a severe sepsis group (25 with severe sepsis and 28, septic shock). Of the 88 people, 19 developed AKI upon or $24 \mathrm{~h}$ after ICU admission, and 17 developed it more than $48 \mathrm{~h}$ after admission, while the remaining 52 were safe from AKI during the observation.

Table 1 provides a general summary of the subjects' conditions. WBC counts and serum PCT levels in the severe sepsis group were higher than that of the sepsis group $(P=0.002, P<0.001)$. There were statistically significant differences in urine sTREM-1 level and disease severity scores, including APACHE II and SOFA scores, among the three groups of patients upon ICU admission $(P<=0.001)$. In terms of kidney function, the $\mathrm{SCr}$ and $\mathrm{BUN}$ levels for the severe sepsis group tended to be higher than sepsis group $(P<0.01)$. The number of severe sepsis patients that required CRRT was greater than for the sepsis group $(P=0.017)$. More patients developed AKI in the severe sepsis group $(P<$ $0.001)$. Regarding the 28 -day survivals, mortality rates for the severe sepsis group were the highest, followed by the sepsis group, and the SIRS group had the lowest rate $(P<0.001)$. Statistically, no significant cross-group difference exists in terms of age, gender, serum CRP, urine output, mechanical ventilation, etiological factors, or accompanying underlying diseases.

\section{Comparisons of urine sTREM-1, WBC counts, serum CRP, and serum PCT levels, and APACHEll scores: for the purpose of early sepsis diagnosis}

On the first day of enrollment, urine sTREM-1, WBC counts, serum PCT and APACHE II scores of the sepsis group had higher values than the SIRS group (Table 1). However, only differences in urine sTREM-1 level and APACHE II score between the groups proved 
Table 1 Clinical and biological data at admission according to the guideline for diagnosis of sepsis

\begin{tabular}{|c|c|c|c|c|}
\hline \multirow[t]{2}{*}{ Characteristics } & \multirow{2}{*}{$\begin{array}{c}\text { SIRS } \\
\mathrm{N}=16\end{array}$} & \multicolumn{3}{|c|}{ Sepsis } \\
\hline & & total $n=88$ & sepsis $n=35$ & severe sepsis $n=53$ \\
\hline Age (years) & $51 \pm 18$ & $60 \pm 19$ & $58 \pm 21$ & $62 \pm 17$ \\
\hline \multicolumn{5}{|l|}{ Gender (n, \%) } \\
\hline Male & $3(19)$ & $23(26)$ & $24(69)$ & $41(77)$ \\
\hline Female & $13(81)$ & $65(74)$ & $11(31)$ & $12(24)$ \\
\hline WBC counts $\left(\times 10^{\wedge} 9 / \mathrm{L}\right)$ & $11.3 \pm 2.8$ & $12.9 \pm 6.7$ & $10.5 \pm 3.9$ & $14.4 \pm 7.7^{\#}$ \\
\hline Serum CRP $(\mathrm{mg} / \mathrm{dl})$ & $11.4(5.9,15.2)$ & $9.5(4.1,17.2)$ & $8.1(2.5,14.7)$ & $11.8(6.1,18.6)$ \\
\hline Serum PCT (ng/ml) & $1.2(0.3,9.5)$ & $2.7(0.4,11.1)$ & $0.4(0.1$ to 2.1$)$ & $4.5(1.2 \text { to } 19.4)^{\#}$ \\
\hline Urine sTREM-1 (pg/ml) & $11.8(3.6,24.1)$ & $61.5(21.3,173.7)^{*}$ & $28.0(10.5,78.8)$ & $83.3(39.6,259.7)^{\#}$ \\
\hline \multicolumn{5}{|l|}{ Renal characteristics } \\
\hline Urinary output (ml/Kg/hr) & - & $1.2 \pm 0.6$ & $1.3 \pm 0.5$ & $1.1 \pm 0.7$ \\
\hline $\mathrm{SCr}(\mu \mathrm{mmol} / \mathrm{L})$ & $83.2(69.25,104.75)$ & $85(60.5,134.4)$ & $65.9(48.0,99.9)$ & $90.8(64.4,167.7)^{\#}$ \\
\hline BUN (mmol/L) & $7.9(4.9,13.2)$ & $10.4(7.5,16.9)$ & $8.0(6.5,10.6)$ & $12.7(8.8(28.9))^{\#}$ \\
\hline APACHE II score & $11.5 \pm 7.6$ & $17.5 \pm 7.5^{*}$ & $13.4 \pm 6.1$ & $20.2 \pm 7.1^{\#}$ \\
\hline SOFA score & - & $8.6 \pm 3.7$ & $5.8 \pm 2.9$ & $10.0 \pm 3.5^{\#}$ \\
\hline MV $(n, \%)$ & $12(75.0)$ & $74(86.0)$ & $29(82.9)$ & $45(84.9)$ \\
\hline CRRT $(n, \%)$ & $2(12.5)$ & $25(28.4)$ & $5(14.3)$ & $20(37.7)^{\#}$ \\
\hline AKI $(n, \%)$ & $2(12.5)$ & $36(39.8)^{*}$ & $4(11.4)$ & $31(58.5)^{\#}$ \\
\hline \multicolumn{5}{|l|}{ Etiological factors (n, \%) } \\
\hline Pulmonary infection & - & $75(85.2)$ & $31(88.6)$ & $44(83.0)$ \\
\hline Abdominal infection & - & $22(25.0)$ & $7(20.0)$ & $15(28.3)$ \\
\hline Urinary tract infection & - & $25(28.4)$ & $13(37.1)$ & $12(22.6)$ \\
\hline Trauma/postoperative infection & - & $33(37.5)$ & $10(28.6)$ & $23(43.4)$ \\
\hline Bacteremia & - & $19(21.6)$ & $9(25.7)$ & $10(18.9)$ \\
\hline Catheter-related infections & - & $13(14.8)$ & $8(22.9)$ & $5(9.4)$ \\
\hline Others & - & $4(4.5)$ & $0(0.0)$ & $4(7.5)$ \\
\hline \multicolumn{5}{|l|}{ Underlying diseases (n, \%) } \\
\hline Hypertension & $5(31.2)$ & $32(332(36.4)$ & $12(34.3)$ & $20(37.7)$ \\
\hline Diabetes & $1(6.2)$ & $14(15.9)$ & $5(14.3)$ & $5(9.4)$ \\
\hline COPD & $1(6.2)$ & $9(10.2)$ & $5(14.3)$ & $4(7.5)$ \\
\hline Coronary heart disease & $4(25.0)$ & $17(19.3)$ & $5(14.3)$ & $12(22.6)$ \\
\hline Immunosuppressed & $0(0.0)$ & $9(10.2)$ & $2(5.7)$ & $7(13.2)$ \\
\hline Nervous system disease & $2(12.5)$ & 12(13.6) & $5(14.3)$ & $7(13.2)$ \\
\hline CKD & $1(6.2)$ & $5(5.7)$ & $1(2.9)$ & $4(7.5)$ \\
\hline Mortality rate $(n, \%)$ & $1(6.2)$ & $41(46.6)^{*}$ & $7(20.0)$ & $34(64.2)^{\#}$ \\
\hline
\end{tabular}

Quantitative data of normal distribution are presented as mean \pm SD. Quantitative data of non-normal distribution are presented as median (25th and 75th percentiles). Qualitative data are presented as $\mathrm{n}(\%) .{ }^{*}$ represents the comparison between SIRS and sepsis, $P<0.05$; \# represents the comparison between sepsis and severe sepsis, $P<0.05$.

AKI, acute kidney injury; APACHE II score, acute physiologic assessment and chronic health evaluation II scores; BUN, blood urea nitrogen; CKD, chronic kidney disease; CRP, C-reactive protein; CRRT, continuous renal replacement treatment; MV, mechanical ventilation; NS, not significant; PCT, procalcitonin; SCr, serum creatinine; SOFA score, sequential organ failure assessment scores; sTREM-1, soluble triggering receptor expressed on myeloid cells-1; WBC, white blood cells.

statistically significant. Figure 1 shows the Receiver Operating Characteristic curves (ROCs) of urine sTREM-1 and APACHE II for sepsis diagnosis.

Dynamic changes in urine sTREM-1, WBC counts, serum CRP and serum PCT levels: for the assessments of the severity and prognosis of sepsis

Figure 2 shows comparisons between the sepsis and severe sepsis groups for urine sTREM-1, WBC counts, serum CRP and serum PCT on days $1,3,5,7,10$, and
14. The differences in urine sTREM-1, WBC counts and serum PCT levels at these six different time points were statistically significant, with the severe sepsis group having all-time higher values, and also showing a higher CRP level on days 5, 7, and 10, which were also statistically significant.

Based on 28-day survivals, the 88 sepsis patients were divided into a survivor group $(\mathrm{n}=47)$ and a non-survivor group $(n=41)$. Figure 3 compares these two groups for their dynamic changes in urine sTREM-1, WBC 

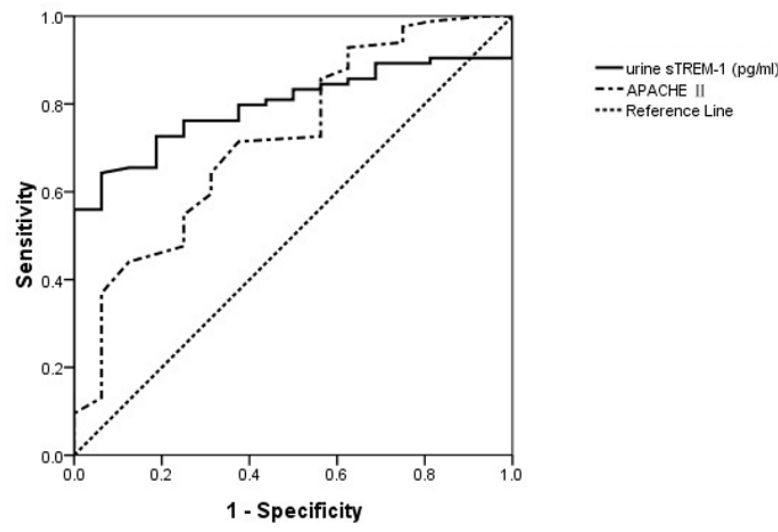
---APACHE II -... Reference Line

Figure 1 ROC curves for urine STREM-1 and APACHEII for distinguishing sepsis from SIRS. Area under the curve (AUC) turned out respectively as urine sTREM-1 $0.797(95 \% \mathrm{Cl} 0.711$ to 0.884) and APACHE II score 0.722 ( $95 \% \mathrm{Cl} 0.586$ to 0.858). Using a cut-off point for urine sTREM-1 of $34.2 \mathrm{pg} / \mathrm{ml}$, the sensitivity was 0.643 , the specificity was 0.938 , Positive Predictive Value (PPV) was 0.983, Negative Predictive Value (NPV) was 0.198 and Youden index (YI) was 0.581. Using a cut-off point for APACHE II score of 13.5, the sensitivity was 0.714 , the specificity was 0.625 , PPV was 0.913 , NPV was 0.197 and YI was 0.339. ROC curves, Receiver Operating Characteristic curves;STREM-1, soluble triggering receptor expressed on myeloid cells-1; APACHEII, acute physiologic assessment and chronic health evaluationll;SIRS, systemic inflammatory response syndrome.

counts, serum CRP, and serum PCT levels. The number of survivors decreased with time $(41,41,32,25,13$ and 7 people on days $1,3,5,7,10$, and 14 respectively). Therefore, for those who died within 14 days, indicator values obtained at the last test before death were substituted for indicator values at later times after death. The curves in Figure 3 show that the non-survivor group had higher urine sTREM-1, WBC counts, serum CRP and serum PCT levels than the survivor group over this period of time. As for non-survivors, their urine sTREM-1 and serum PCT levels increased with the passage of time, while their WBC counts and serum CRP levels tended to decline. The survivor group exhibited no obvious changes in urine sTREM-1 levels, while their WBC counts, serum CRP, and serum PCT levels were obviously on the decline.

\section{Urine sTREM-1: diagnostic value for AKI}

Of the 88 severe sepsis cases, 19 developed AKI upon or within $24 \mathrm{~h}$ of their ICU admission, and 17 developed AKI $48 \mathrm{~h}$ later. The other 52 did not develop AKI complications during the time of observation. Table 2 shows the results for the 17 AKI patients who developed AKI before $48 \mathrm{~h}$ diagnosis of AKI, such as urine sTREM-1, urine output, $\mathrm{CCr}, \mathrm{SCr}$ and BUN. Table 2 also shows these indicators' levels upon ICU admission for the 52 patients that did not develop complications. Comparisons were made between these two groups of patients. The AKI patients' urine sTREM-1 levels at $48 \mathrm{~h}$ before diagnosis were significantly higher than those for the non- AKI patients $(P<0.001)$. The AKI patients' $\mathrm{CCr}$, $\mathrm{SCr}$ and BUN levels at $48 \mathrm{~h}$ before diagnosis were also higher than for the non-AKI patients $(P<0.05)$.

Multivariate logistic regression was used to assess possible risk factors for AKI occurrence. The variables taken into consideration here include severity of sepsis, urine output, CCr, SCR, BUN, urine sTREM-1 and a history of chronic kidney disease. First, from univariate analyses, four variables were selected for multivariate regression: severity, CCr, SCR and urine sTREM-1. Finally, only urine STREM-1 and severity were entered into the regression equation. Urine sTREM-1 with a regression coefficient $=$ 0.02 , OR $=1.02$ and Wald coefficient $=5.246(P=0.022)$. Severity with a regression coefficient $=2.074, \mathrm{OR}=7.956$ and Wald coefficient $=5.204(P=0.023)$.

Figure 4 shows ROC curves for AKI diagnosis for urine sTREM-1, SCR, and BUN levels. The corresponding AUCs were as Table 3. Using a cut-off point of $69.04 \mathrm{pg} / \mathrm{ml}$ for sTREM-1, the sensitivity turns out 0.941 and the specificity, 0.760 .

\section{Discussion}

Sepsis is a quite common cause of critical diseases and complications, and one of the risk factors for AKI and MODS [28]. When an infection occurs, lipopolysaccharides, lipopeptides, microbial DNA, peptide polysaccharides, lipoteichoic acid and other substances can trigger innate immune reactions mediated by Toll-like receptors (TLRs), NOD-like receptors (NLRs), and other relevant molecules, such as MyD88, and so on. These can subsequently engender both humoral and cellular immune responses. Through the actions of various signals, numerous cytokines, metabolic products of arachidonic acid, leukotrienes, thrombosis-forming substances and inflammatory mediators are produced. In addition, complement and coagulation pathways are activated $[29,30]$. In case of sepsis, systemic inflammatory factor-induced immune reactions, such as innate and adaptive immune responses, play a critical role in the induction and development of organ dysfunctions, such as AKI $[23,31]$. This may result from increased expressions of TLRs and NLRs on renal tubule epithelia as well as local endothelial cells inside the kidney. This can cause localized inflammatory reactions in the kidney and oxidative stress, as well as inducing epithelial and endothelial cell injury, and induce changes in the glomerular pore diameter and charge barrier [24,32]

Our study shows that urine sTREM-1 has a positive predictive value for the early diagnosis of sepsis. We do 

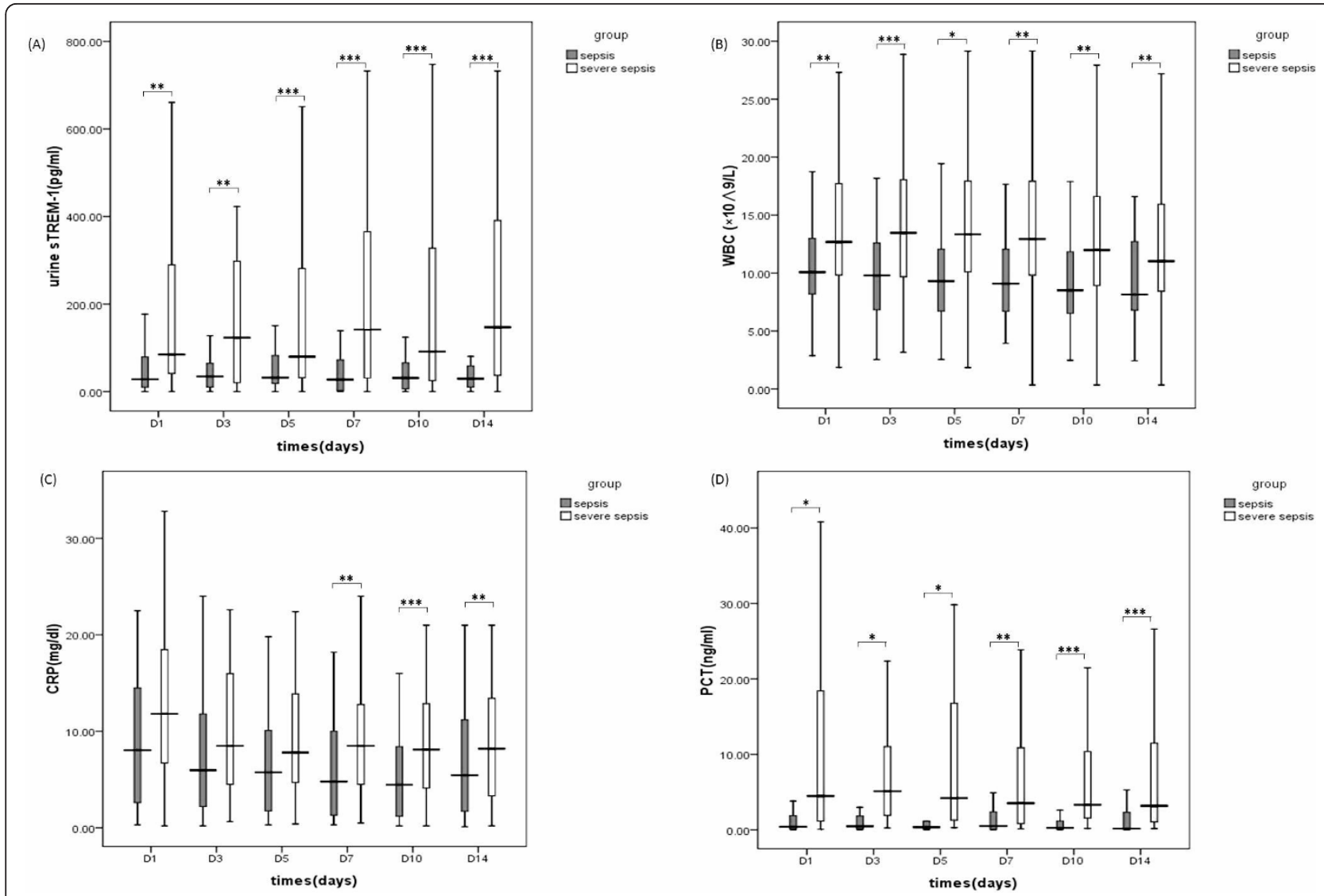

Figure 2 sTREM-1 (A), WBC (B), CRP (C), and PCT (D) levels measured over 14 days at different stages of sepsis.. ${ }^{*} P<0.05$, ${ }^{* *} P<0.01,{ }^{* * *} P<$ 0.001. CRP, C-reactive protein; PCT, procalcitonin; sTREM-1, soluble triggering receptor expressed on myeloid cells-1; WBC, White blood cells.

find that there are statistically significant differences between SIRS and sepsis patients upon their ICU admission in urine sTREM-1 level and APACHE II score. ROC curves analysis displays that the urine STREM-1 AUC is conspicuously higher than any other indicator and its sensitivity reaches 0.643 .

While conducting dynamic assessments of the severity and prognosis of sepsis, we found that a severe sepsis group exhibited higher urine sTREM-1, WBC, and serum PCT level than a sepsis group on days $1,3,5,7$, 10 , and 14, reckoned from ICU admission $(P<0.05)$. All this points to the value of urine STREM-1, WBC, and serum PCT, as biomarkers, in the discrimination of severe (severe sepsis + septic shock) accompanied by organ dysfunction. Moreover, the differences in these indices between the two groups were observed even on the first day of ICU admission, which is conducive to an early diagnosis of severe sepsis and a timely intervention.

During the 14 days of observation, the non-survivors' urine sTREM-1 levels continued to climb with the passage of time, while the survivors' remained relatively lower. This suggests that urine sTREM-1 may have connections with the prognosis of the disease, and that its continuous, relatively lower expression indicates that the inflammatory response has been brought under control and prognosis might be better. In contrast, in the case of non-survivors, the sTREM-1 level, possibly owing to positive feedback provided by downstream inflammatory factors, rises continually $[33,34]$. Because of extracellular partial exfoliation brought about by metalloproteinase proteolysis, TREM-1 gives rise to its $27 \mathrm{kDa}$ soluble protein sTREM-1 [15,35], which suggests the release of additional pro-inflammatory cytokines and mediators as well as a continuous or progressive overactive inflammatory response and a poor prognosis. The non-survivors' WBC counts, serum CRP, and serum PCT levels measured higher than those of the survivors during the corresponding period, suggesting a poor prognosis. However, the non-survivors' WBC count and serum CRP level tended to go down towards the end of the course of the disease. All this suggests that the dynamic changes in urine sTREM-1 and serum PCT may well reflect the body's inflammatory responses and the prognosis of sepsis. These two indicators provide more ideal medical tips than WBC counts and serum 
(A)

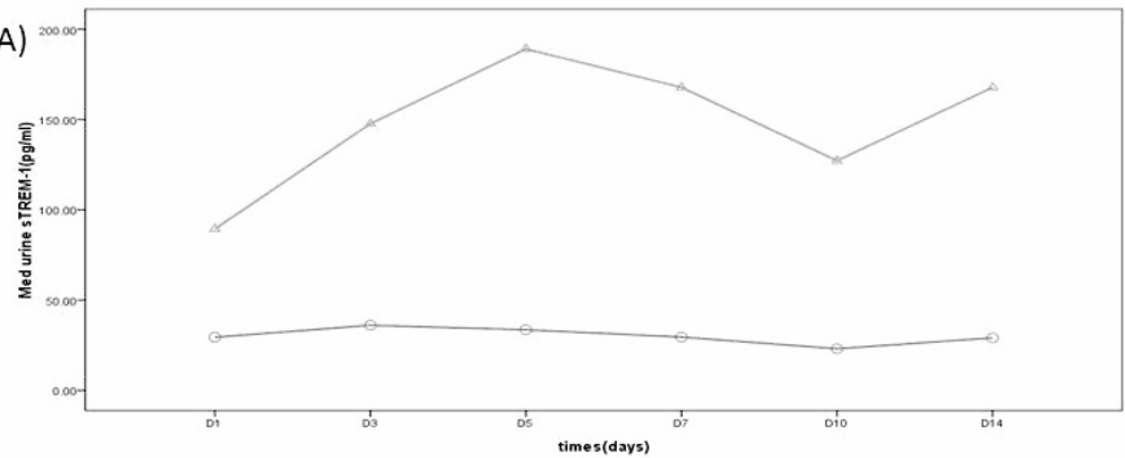

(B)

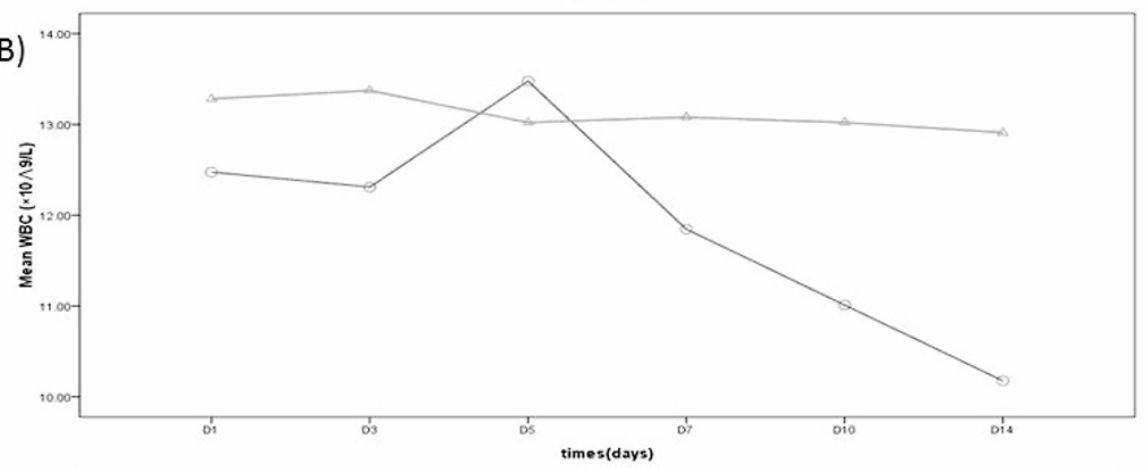

(C)

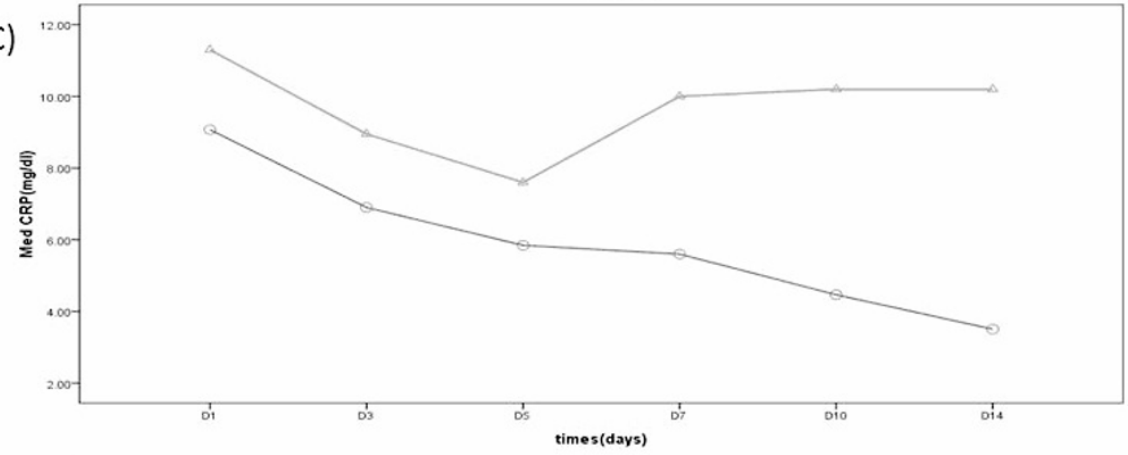

(D)

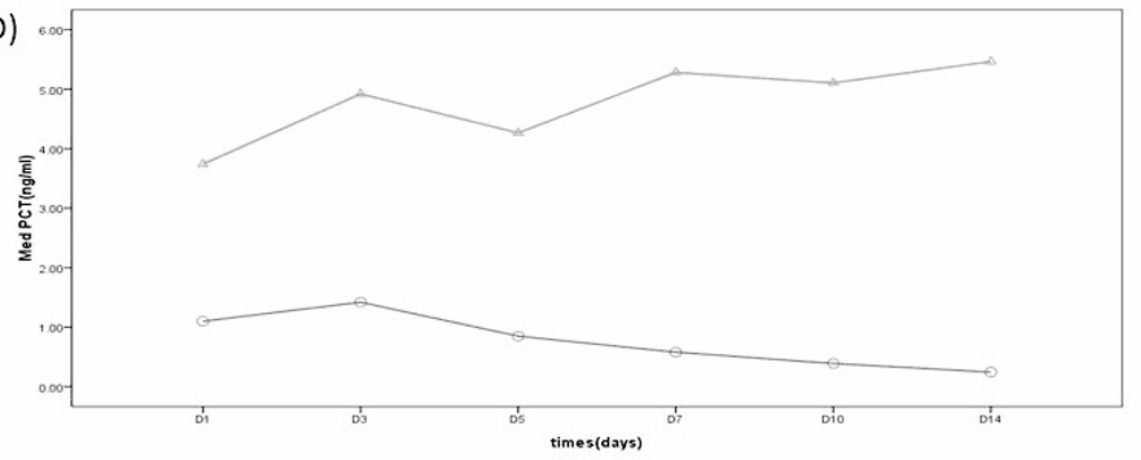

outcome

survivor

$\triangle$ non-survivor

outcome

survivor

$\triangle$ non-survivor

outcome

survivor

$\triangle$ non-survivor

outcome

survivor

$\triangle$ non-survivor

Figure 3 sTREM-1 (A), WBC (B), CRP (C), and PCT (D) levels measured over 14 days based on 28-day survival. The differences of patients diagnosed with sepsis in urine sTREM-1 and serum PCT levels at these six different time points were statistically significant $(P<0.05)$, with the non-survivors group having higher values at all time points, and also showing a higher CRP level on days 7, 10 and 14, which were also statistically significant $(P<0.05)$. WBC counts in the non-survivors group were also higher than that of the survivors group, but with no apparent difference between them at all time points. CRP, C-reactive protein; PCT, procalcitonin; sTREM-1, soluble triggering receptor expressed on myeloid cells-1; WBC, white blood cells. 
Table 2 Comparison diagnostic value of sTREM-1, urinary output, CCr, SCr, BUN levels for AKI before 48 hours

\begin{tabular}{llll}
\hline \multicolumn{1}{c}{ Parameters } & AKI & Non- AKI & P-value \\
\hline & $\mathrm{N}=17$ & $\mathrm{~N}=52$ & $<0.001$ \\
urine sTREM-1 (median-25th and 75th percentiles, $\mathrm{pg} / \mathrm{ml})$ & $288.74(108.65,524.07)$ & $35.66(7.92,75.73)$ & 0.086 \\
urinary output (mean \pm SD, $\mathrm{ml} / \mathrm{Kg} / \mathrm{hr})$ & $1.28 \pm 0.62$ & $1.62 \pm 0.70$ & 0.002 \\
CCr (mean \pm SD, $\mathrm{ml} /$ minute) & $55.59 \pm 30.95$ & $104.28 \pm 60.94$ & 0.001 \\
Scr (median-25th and 75th percentiles, $\mu \mathrm{mmol} / \mathrm{L})$ & $105.0(71.7,176.15)$ & $65.0(52.18,90.98)$ & 0.038 \\
BUN (median-25th and 75th percentiles, mmol/L) & $17.53(8.02,29.73)$ & $8.97(6.66,12.43)$ & \\
\hline
\end{tabular}

sTREM-1, soluble triggering receptor expressed on myeloid cells-1;CCr, creatinine clearance; SCr, serum creatinine; BUN, blood urea nitrogen; AKI, acute kidney injury.

CRP, as progressive rise of urine sTREM-1 and serum PCT levels may signify a bad prognosis

We discovered that $48 \mathrm{~h}$ before AKI occurrence, in contrast with a non-AKI group, that AKI patients had an obvious increase in urine sTREM-1 $(P<0.001)$. The AUC for an ROC curve was 0.922 (95\% CI: 0.850 to 0.995 ), sensitivity was $0.941 \%$ (based on a cut-off point of $69.04 \mathrm{pg} / \mathrm{ml}$ ), and specificity, 0.76. Although the AKI group exhibited higher $\mathrm{SCr}$ and BUN level than the nonAKI group before AKI occurrence, a sharp rise was observed only after AKI occurrence. It, therefore, can be deduced that urine sTREM-1 has its value for early sepsis diagnosis, while the indices of SCR and BUN are more significant for assessing the progress of AKI. For our multivariate logistic regression, urine sTREM-1 and sepsis severity were factors entered into the final regression equation, which suggests that throughout the study period, urine sTREM-1 and severity are to be regarded as

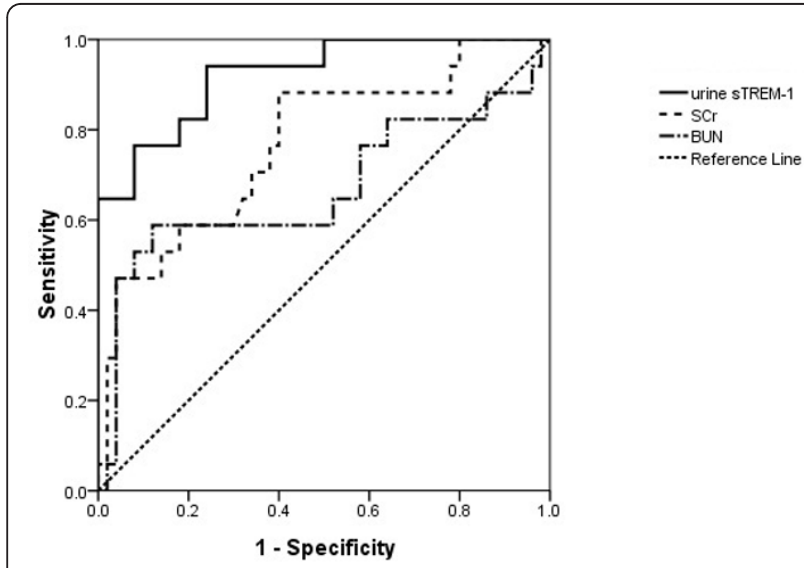

Figure 4 ROC curves for urine STREM-1, SCr, BUN level for diagnosing AKI before $\mathbf{4 8}$ hours. ROC curves, Receiver Operating Characteristic curves;STREM-1, soluble triggering receptor expressed on myeloid cells-1; SCR, serum creatinine; BUN, blood urea nitrogen; AKI, acute kidney injury. possible risk factors and urine sTREM-1 possesses potential significance for the assessment of kidney function.

The present study also has some limitations. (1) The sample size was not sufficiently large, including only 17 cases with AKI diagnosed $48 \mathrm{~h}$ after ICU admission. For this reason, no statistical significance was found with regards to whether urine STREM-1 has relevance to AKIN classification (no data reported in this regard) [27]. (2) Blood and urine samples were collected every other day, and not on a day-to-day basis. Therefore, no assessments could be made of kidney function $24 \mathrm{~h}$ before AKI occurrence. It cannot be ruled out that renal function decline already existed before AKI diagnosis. (3) There are reports that there are no obvious changes in urine sTREM-1 levels with urinary tract infections [36]. It is, therefore, predicted that a rise in urine sTREM- 1 is an inflammatory factor-induced localized kidney immune response brought on by sepsis, not solely by localized inflammatory responses [23,31]. On account of this, the present study does not involve cases with urinary tract infection. (4) In our study, the average CRRT duration for AKI patients measures $11.6 \pm 3.8 \mathrm{~h}$ per day. In virtue of not receiving continuously renal replacement therapy, the AKI patients in our study might still be featured with a larger quantity of inflammatory factors in their body. In consideration of the sample size, we did not exclude those who received CRRT from this study.

\section{Conclusions}

Urine sTREM-1 may play a role in the early diagnosis of sepsis. The dynamic change in urine sTREM-1 may be an aid to distinguish severity of sepsis, and be more accurate and sensitive than traditional indicators for the dynamic assessments of prognosis. We find that urine sTREM-1 has significance in the early diagnosis of sepsis-related AKI, and could likely become a new marker for such injuries. Owing to the limitations of our sample size, prospective clinical studies are still wanted to provide further proof for the clinical diagnostic value of urine sTREM-1. In addition, further studies are to be expected on the role and mechanisms of urine sTREM-1 for AKI. 
Table 3 Area under ROC curves as means of diagnosing AKI before 48 hours

\begin{tabular}{|c|c|c|c|c|c|c|c|c|c|c|c|}
\hline Variable & AUC & Std. Error & $P$-value & Asymptotic $95 \%$ & dence Interval & Cut & sen & S pe & PPV & NPV & YI \\
\hline & & & & Lower limit & Upper limit & point & & & & & \\
\hline Urine sTREM-1 & 0.922 & 0.037 & $<0.001$ & 0.85 & 0.994 & 69.04 & 0.941 & 0.76 & 0.656 & 0.964 & 0.701 \\
\hline $\mathrm{CCr}$ & 0.212 & 0.061 & $<0.001$ & 0.093 & 0.331 & - & - & - & - & - & - \\
\hline $\mathrm{SCr}$ & 0.776 & 0.068 & 0.001 & 0.644 & 0.909 & 68.3 & 0.882 & 0.6 & 0.517 & 0.911 & 0.482 \\
\hline BUN & 0.685 & 0.091 & 0.024 & 0.507 & 0.864 & 15.23 & 0.588 & 0.88 & 0.704 & 0.815 & 0.468 \\
\hline
\end{tabular}

ROC curves, receiver operating characteristic curves; AKI, acute kidney injury;BUN, blood urea nitrogen; CCr, creatinine clearance; SCr, serum creatinine; sTREM-1, soluble triggering receptor expressed on myeloid cells-1.

\section{Key messages}

- Identification of infection on classic biomarkers is insufficient. But urine sTREM-1 may turn out the most reliable marker for sepsis and its severity.

- Dynamic changes in urine sTREM-1 are more accurate and sensitive than traditional indicators for the dynamic assessments of severity and prognosis of sepsis.

- Urine sTREM-1 can also provide an early warning of possible secondary AKI in sepsis patients. It is likely to be a risk factor and has potential significance for the assessment of kidney function.

- Urine sTREM-1 is likely to become a biomarker with optimistic prospects of clinical application.

\section{Abbreviations}

AKI: acute kidney injury; AKIN: Acute Kidney Injury Network; APACHE II score: Acute Physiologic Assessment and Chronic Health Evaluation II scores; AUC: areas under curve; BUN: blood urea nitrogen; $\mathrm{CCr}$ : creatinine clearance; $\mathrm{Cl}$ : confidence interval; CKD: chronic kidney disease; CRP: C-reactive protein; CRRT: continuous renal replacement treatment; MODS: multiple organ dysfunction syndrome; MV: mechanical ventilation; NLRs: NOD-like receptors; PCT: procalcitonin; ROC curves: Receiver Operating Characteristic curves; SCr: serum creatinine; SIRS: systemic inflammatory response syndrome; SOFA score: Sequential Organ Failure Assessment scores; sTREM-1: soluble triggering receptor expressed on myeloid cells-1; TLRs: Toll-like receptors; WBC: white blood cells.

\section{Acknowledgements}

Written consent for publication was obtained from the patient or their relatives.

We express our thanks to the staff members of the RICU, CCM, and EICU of the CPLA General Hospital for their assistance in this study. This study was supported by the Chinese National Science and Technology Pillar Program (NO: 2009BAl86B03). The funders had no role in the study design, data collection and analysis, decision to publish, or preparation of the manuscript.

\section{Author details}

${ }^{1}$ Department of Respiratory Diseases, Chinese PLA General Hospital, 28 Fuxing Rd, Beijing, 100853, China. ${ }^{2}$ Department of Medical Statistics, Chinese PLA General Hospital, 28 Fuxing Rd, Beijing, 100853, China. ${ }^{3}$ Medical School, Nankai University, 94 Weijin Rd, Tianjin, 300071, China.

\section{Authors' contributions}

LS designed the study, carried it out in the SICU, performed the data analysis, and wrote the first manuscript draft. LF and PY carried out the study in the EICU and the RICU, respectively. JZ and YJ conceived the initial idea for using STREM-1 levels for infectious diseases and supplemented the study design. DF guided the data analysis and the use of medical statistics. LX was responsible for protocol revisions, data analysis, and final draft revision. All authors have read and approved the final manuscript for publication.

\section{Competing interests}

The authors declare that they have no competing interests.

Received: 6 June 2011 Revised: 25 August 2011

Accepted: 24 October 2011 Published: 24 October 2011

\section{References}

1. Martin GS, Mannino DM, Eaton S, Moss M: The epidemiology of sepsis in the United States from 1979 through 2000. N Engl J Med 2003, 348:1546-1554.

2. Vincent $J$, Nelson DR, Williams MD: Is worsening multiple organ failure the cause of death in patients with severe sepsis? Crit Care Med 2011, 39:1050-1055.

3. Hoste EA, Clermont G, Kersten A, Venkataraman R, Angus DC, De Bacquer D, Kellum JA: RIFLE criteria for acute kidney injury are associated with hospital mortality in critically ill patients: a cohort analysis. Crit Care 2006, 10:R73.

4. Steinvall I, Bak Z, Sjoberg F: Acute kidney injury is common, parallels organ dysfunction or failure, and carries appreciable mortality in patients with major burns: a prospective exploratory cohort study. Crit Care 2008, 12:R124.

5. Lameire N, Van Biesen W, Vanholder R: Acute renal failure. Lancet 2005, 365:417-430

6. Otero RM, Nguyen HB, Huang DT, Gaieski DF, Goyal M, Gunnerson K, Trzeciak S, Sherwin R, Holthaus CV, Osborn T, Rivers EP: Early goal-directed therapy in severe sepsis and septic shock revisited: concepts, controversies, and contemporary findings. Chest 2006, 130:1579-1595.

7. Bouchon A, Facchetti F, Weigand MA, Colonna M: TREM-1 amplifies inflammation and is a crucial mediator of septic shock. Nature 2001, 410:1103-1107.

8. Gibot S, Kolopp-Sarda MN, Bene MC, Cravoisy A, Levy B, Faure GC, Bollaert PE: Plasma level of a triggering receptor expressed on myeloid cells-1: its diagnostic accuracy in patients with suspected sepsis. Ann Intern Med 2004, 141:9-15.

9. Gibot S, Cravoisy A, Levy B, Bene MC, Faure G, Bollaert PE: Soluble triggering receptor expressed on myeloid cells and the diagnosis of pneumonia. N Engl J Med 2004, 350:451-458.

10. Liu CL, Hsieh WY, Wu CL, Kuo HT, Lu YT: Triggering receptor expressed on myeloid cells- 1 in pleural effusions: a marker of inflammatory disease. Respir Med 2007, 101:903-909.

11. Collins CE, La DT, Yang HT, Massin F, Gibot S, Faure G, Stohl W: Elevated synovial expression of triggering receptor expressed on myeloid cells 1 in patients with septic arthritis or rheumatoid arthritis. Ann Rheum Dis 2009, 68:1768-1774.

12. Determann RM, Weisfelt M, de Gans J, van der Ende A, Schultz MJ, van de Beek D: Soluble triggering receptor expressed on myeloid cells $1:$ a biomarker for bacterial meningitis. Intensive Care Med 2006, 32:1243-1247.

13. Kusanovic JP, Romero R, Chaiworapongsa T, Mittal P, Mazaki-Tovi S, Vaisbuch E, Erez O, Gotsch F, Than NG, Edwin SS, Pacora P, Jodicke C, Yeo L, Hassan SS: Amniotic fluid sTREM-1 in normal pregnancy, spontaneous parturition at term and preterm, and intra-amniotic infection/inflammation. J Matern Fetal Neonatal Med 2010, 23:34-47.

14. Determann RM, van Till JW, van Ruler O, van Veen SQ, Schultz MJ, Boermeester MA: sTREM-1 is a potential useful biomarker for exclusion of ongoing infection in patients with secondary peritonitis. Cytokine 2009, 46:36-42.

15. Gibot S, Kolopp-Sarda MN, Bene MC, Bollaert PE, Lozniewski A, Mory F, Levy B, Faure GC: A soluble form of the triggering receptor expressed on 
myeloid cells-1 modulates the inflammatory response in murine sepsis. $J$ Exp Med 2004, 200:1419-1426.

16. Dimopoulou I, Orfanos SE, Pelekanou A, Kotanidou A, Livaditi O, Augustatou C, Zervou M, Douka E, Theodorakopoulou M, Karagianni V, Douzinas E, Armaganidis A, Giamarellos-Bourboulis EJ: Serum of patients with septic shock stimulates the expression of Trem-1 on U937 monocytes. Inflamm Res 2009, 58:127-132.

17. Zhang J, She D, Feng D, Jia Y, Xie L: Dynamic changes of serum soluble triggering receptor expressed on myeloid cells-1 (sTREM-1) reflect sepsis severity and can predict prognosis: a prospective study. BMC Infect Dis 2011, 11:53.

18. Gibot S, Cravoisy A, Kolopp-Sarda MN, Bene MC, Faure G, Bollaert PE, Levy B: Time-course of STREM (soluble triggering receptor expressed on myeloid cells)-1, procalcitonin, and C-reactive protein plasma concentrations during sepsis. Crit Care Med 2005, 33:792-796.

19. Friedewald JJ, Rabb H: Inflammatory cells in ischemic acute renal failure. Kidney Int 2004, 66:486-491.

20. Bonventre JV, Zuk A: Ischemic acute renal failure: an inflammatory disease? Kidney Int 2004, 66:480-485.

21. Schrier RW: Pathophysiology of ischemic acute renal injury. In Diseases of the Kidney and Urinary Tract. Volume 2.. 8 edition. Philadelphia, PA, USA: Lippincott Williams \& Wilkins; 2007.

22. Schrier RW: ARF: pathogenesis, diagnosis andmanagement. Renal and Electrolyte Disorders. 6 edition. Philadelphia, PA, USA: Lippincott Williams \& Wilkins; 2003.

23. Kinsey GR, Li L, Okusa MD: Inflammation in acute kidney injury. Nephron Exp Nephrol 2008, 109:e102-107.

24. Akcay A, Nguyen Q, Edelstein CL: Mediators of inflammation in acute kidney injury. Mediators Inflamm 2009, 2009:137072.

25. Bone RC, Balk RA, Cerra FB, Dellinger RP, Fein AM, Knaus WA, Schein RM, Sibbald WJ: Definitions for sepsis and organ failure and guidelines for the use of innovative therapies in sepsis. The ACCP/SCCM Consensus Conference Committee. American College of Chest Physicians/Society of Critical Care Medicine. Chest 1992, 101:1644-1655.

26. Levy MM, Fink MP, Marshall JC, Abraham E, Angus D, Cook D, Cohen J, Opal SM, Vincent JL, Ramsay G: 2001 SCCM/ESICM/ACCP/ATS/SIS International Sepsis Definitions Conference. Crit Care Med 2003, 31:1250-1256.

27. Mehta RL, Kellum JA, Shah SV, Molitoris BA, Ronco C, Warnock DG, Levin A: Acute Kidney Injury Network: report of an initiative to improve outcomes in acute kidney injury. Crit Care 2007, 11:R31.

28. Uchino S, Kellum JA, Bellomo R, Doig GS, Morimatsu H, Morgera S, Schetz M, Tan I, Bouman C, Macedo E, Gibney N, Tolwani A, Ronco C, Beginning and Ending Supportive Therapy for the Kidney (BEST Kidney) Investigators: Acute renal failure in critically ill patients: a multinational, multicenter study. JAMA 2005, 294:813-818.

29. Zhang B, Ramesh G, Uematsu S, Akira S, Reeves WB: TLR4 signaling mediates inflammation and tissue injury in nephrotoxicity. J Am Soc Nephrol 2008, 19:923-932.

30. Goncalves GM, Zamboni DS, Camara NO: The role of innate immunity in septic acute kidney injuries. Shock 2010, 34(Suppl 1):22-26.

31. Ishikawa K, May CN, Gobe G, Langenberg C, Bellomo R: Pathophysiology of septic acute kidney injury: a different view of tubular injury. Contrib Nephrol 2010, 165:18-27.

32. El-Achkar TM, Hosein M, Dagher PC: Pathways of renal injury in systemic gram-negative sepsis. Eur J Clin Invest 2008, 38(Suppl 2):39-44.

33. Colonna M: TREMs in the immune system and beyond. Nat Rev Immunol 2003, 3:445-453.

34. Ornatowska M, Azim AC, Wang X, Christman JW, Xiao L, Joo M, Sadikot RT: Functional genomics of silencing TREM-1 on TLR4 signaling in macrophages. Am J Physiol Lung Cell Mol Physiol 2007, 293:L1377-1384.

35. Gomez-Pina V, Soares-Schanoski A, Rodriguez-Rojas A, Del Fresno C, Garcia F, Vallejo-Cremades MT, Fernandez-Ruiz I, Arnalich F, Fuentes-Prior P, Lopez-Collazo E: Metalloproteinases shed TREM-1 ectodomain from lipopolysaccharide-stimulated human monocytes. J Immunol 2007, 179:4065-4073.
36. Determann RM, Schultz MJ, Geerlings SE: Soluble triggering receptor expressed on myeloid cells- 1 is not a sufficient biological marker for infection of the urinary tract. J Infect 2007, 54:e249-250.

doi:10.1186/cc10508

Cite this article as: Su et al.: Diagnostic value of urine STREM-1 for sepsis and relevant acute kidney injuries: a prospective study. Critical Care 2011 15:R250

\section{Submit your next manuscript to BioMed Central and take full advantage of:}

- Convenient online submission

- Thorough peer review

- No space constraints or color figure charges

- Immediate publication on acceptance

- Inclusion in PubMed, CAS, Scopus and Google Scholar

- Research which is freely available for redistribution

Submit your manuscript at www.biomedcentral.com/submit 